\title{
Higher-order saddlepoint approximations in the Vasicek portfolio credit loss model
}

\author{
Xinzheng Huang \\ Delft Institute of Applied Mathematics, Delft University of Technology, Mekelweg 4, \\ 2628 CD, Delft, The Netherlands \\ and \\ Group Risk Management, Rabobank, Croeselaan 18, 3521 CB, Utrecht, The Netherlands; \\ email: x.huang@ewi.tudelft.nl
}

\section{Cornelis W. Oosterlee}

Delft Institute of Applied Mathematics, Delft University of Technology, Mekelweg 4, 2628 CD, Delft, The Netherlands

and

CWI-National Research Institute for Mathematics and

Computer Science, Kruislaan 413, 1098 SJ, Amsterdam, The Netherlands;

email: c.w.oosterlee@math.tudelft.nl

\section{Hans van der Weide}

Delft Institute of Applied Mathematics, Delft University of Technology, Mekelweg 4, 2628 CD, Delft, The Netherlands; email: j.a.m.vanderweide@ewi.tudelft.nl

This paper utilizes the saddlepoint approximation as an efficient tool to estimate the portfolio credit loss distribution in the Vasicek model. Value-atrisk(VaR), the risk measure chosen in the Basel II Accord for the evaluation of capital requirement, can then be found by inverting the loss distribution. The VaR contribution (VaRC), expected shortfall (ES) and ES contribution (ESC) can all be calculated accurately. Saddlepoint approximation is well known to provide good approximations to very small tail probabilities, which makes it a very suitable technique in the context of portfolio credit loss. The portfolio credit model we employ is the Vasicek one-factor model, which has an analytical solution if the portfolio is well diversified. The Vasicek asymptotic formula fails, however, when the portfolio is dominated by a few loans much larger than the rest. We show that saddlepoint approximation is able to handle such exposure concentration. We also point out that the saddlepoint approximation technique can be readily applied to more general Bernoulli mixture models (possibly multi-factor). It can further handle portfolios with random loss given default (LGD).

We are grateful to Dr Mâcé Mesters and Dr Sacha van Weeren from Rabobank for drawing our attention to this issue and for their helpful comments. We would also like to thank the participants at the first AMAMEF conference for valuable feedback. 


\section{INTRODUCTION}

The integral issues in portfolio credit loss modeling are the determination of the value-at-risk ( $\mathrm{VaR}$ ) and the $\mathrm{VaR}$ contribution (VaRC). Value-at-risk is the risk measure chosen in the Basel II Accord (Basel Committee on Bank Supervision 2005) for the evaluation of capital requirement. The VaRC measures how much each obligor in a portfolio contributes to the total VaR. It is equally as important as $\mathrm{VaR}$ because it is necessary for loan pricing and it can provide limits on large credit exposures. It may also be useful for profitability assessment, asset allocation and portfolio optimization. There are several forms of risk contribution in the literature and we adopt the marginal contribution given by Gourieroux et al (2000), which is the sensitivity of the risk to an infinitesimal fractional change in exposure.

The Vasicek (2002) portfolio credit loss model is among the most popular models quantifying portfolio credit risk. In particular, it is the basis of the Basel II internal-ratings-based (IRB) approach. The Vasicek model is a one-period defaultmode model, ie, loss only occurs when an obligor defaults in a fixed time horizon. Under certain homogeneity conditions, the Vasicek one-factor model leads to very simple analytic asymptotic approximation of loss distribution and the VaR. The approximation works very well when the portfolio is large and there is no exposure concentration present, ie, the portfolio is not dominated by a few loans. However, the Vasicek one-factor model cannot detect exposure concentration when it is inherent in the portfolio as it then tends to underestimate risk.

This paper utilizes the saddlepoint approximation as an efficient tool to estimate portfolio credit loss distribution. The saddlepoint approximation method is well known to provide good approximations to very small tail probabilities, which makes it a very suitable technique in the context of portfolio credit loss. The use of saddlepoint approximation in portfolio credit loss was pioneered in a series of articles by Martin et al (2001a, b). Gordy (2002) showed that saddlepoint approximation is fast and robust when applied to CreditRisk ${ }^{+}$. The approach in this paper is different in that (i) we employ the saddlepoint approximation in the Vasicek model and (ii) we apply the saddlepoint approximation to the conditional moment generating function (MGF) of portfolio loss $L$ rather than to the unconditional MGF. We show that this change in implementation of the saddlepoint approximation leads to very accurate results on the portfolio loss distribution, the VaR and VaRC, even for small portfolios and portfolios with exposure concentration. In addition to the VaR and VaRC, we also give the saddlepoint approximations for the Expected Shortfall (ES) and ES contribution (ESC).

The rest of the article is organized as follows. In Section 2 we introduce the popular risk measures and risk contributions and we review the Vasicek onefactor model. Section 3 gives a brief introduction to saddlepoint approximation and Section 4 describes how it can be used in the context of portfolio credit loss modeling. Numerical results are presented in Section 5. We mainly work with the Vasicek one-factor model, but also include an example of a Gaussian multi-factor 
model. Section 6 extends the use of the saddlepoint approximation to more general situations. Conclusions are given in Section 7.

\section{PORTFOLIO CREDIT LOSS MODELING}

\subsection{Risk measures and risk contributions}

Consider a portfolio consisting of $n$ obligors. Any obligor $i$ can be characterized by three constant quantities: the probability of default $\mathrm{PD}_{i}$, the exposure at default $\mathrm{EAD}_{i}$ and the loss given default $\mathrm{LGD}_{i}$. Obligor $i$ is subject to default after a fixed time horizon and the default can be modeled as a Bernoulli random variable $D_{i}$ such that:

$$
D_{i}= \begin{cases}1 & \text { with probability } \mathrm{PD}_{i} \\ 0 & \text { with probability } 1-\mathrm{PD}_{i}\end{cases}
$$

EAD measures the amount of the facility that is likely to be drawn if a default occurs and LGD is the proportion of the EAD that will be lost if a default occurs. To simplify notation, it is possible to define the effective exposure of obligor $i$ by $w_{i}=\mathrm{EAD}_{i} \times \mathrm{LGD}_{i}$, then the loss incurred by the obligor $i$ is given by:

$$
L_{i}=\mathrm{EAD}_{i} \times \mathrm{LGD}_{i} \times D_{i}=w_{i} D_{i}
$$

It follows that the portfolio loss is given by:

$$
L=\sum_{i=1}^{n} L_{i}=\sum_{i=1}^{n} w_{i} D_{i}
$$

Let $\alpha$ be some given confidence level, the $\alpha$-quantile of the loss distribution of $L$ in this context is VaR. Thus:

$$
\operatorname{VaR}_{\alpha}=\inf \{x: \mathbb{P}(L \leq x) \geq \alpha\}
$$

Usually the $\alpha$ of interest is close to 1 . VaR is the risk measure chosen in the Basel II Accord (Basel Committee on Bank Supervision 2005) for the evaluation of capital requirement, which means that a bank that complies with Basel II needs to reserve capital of size $\mathrm{VaR}_{\alpha}$ as a cushion for extreme losses. However, it is known that $\mathrm{VaR}$ is not coherent and, in particular, not subadditive (see Artzner et al 1999). So we also consider ES, a coherent alternative to the VaR. It is defined as the conditional expectation of the loss given that the loss exceeds the VaR:

$$
\mathrm{ES}_{\alpha}=E\left[L \mid L \geq \mathrm{VaR}_{\alpha}\right]
$$

A risk contribution measures how much each obligor in a portfolio contributes to the total risk. This is equally as important as risk measures because it is necessary for loan pricing and it can provide limits on large credit exposures. A desirable property of the risk contributions is that they sum up to the corresponding risk measure, for practical purposes such as profitability assessment, economic capital allocation and portfolio optimization. For example, we want the VaRCs to 
add up to total VaR, ie:

$$
\sum_{i=1}^{n} \mathrm{VaRC}_{i}=\mathrm{VaR}
$$

A common measure of risk contribution that satisfies this property is the sensitivity of the risk to an infinitesimal fractional change in exposure, as given by Gourieroux et al (2000). Under some continuity conditions, the VaRC coincides with the conditional expectation of $L_{i}$ given that the portfolio loss $L$ takes the value $\operatorname{VaR}_{\alpha}(L)$, ie:

$$
\operatorname{VaRC}_{i, \alpha}=w_{i} \frac{\partial \operatorname{VaR}_{\alpha}}{\partial w_{i}}(L)=w_{i} E\left[D_{i} \mid L=\operatorname{VaR}_{\alpha}(L)\right]
$$

The sum of the VaRCs is indeed equal to the total VaR, ie:

$$
\begin{aligned}
\sum_{i=1}^{n} w_{i} E\left[D_{i} \mid L=\operatorname{VaR}_{\alpha}(L)\right] & =E\left[\sum_{i=1}^{n} L_{i} \mid L=\operatorname{VaR}_{\alpha}(L)\right] \\
& =E\left[L \mid L=\operatorname{VaR}_{\alpha}(L)\right] \\
& =\operatorname{VaR}_{\alpha}(L)
\end{aligned}
$$

Similarly, the ESC is given by:

$$
w_{i} \frac{\partial \mathrm{ES}_{\alpha}}{\partial w_{i}}(L)=w_{i} E\left[D_{i} \mid L \geq \operatorname{VaR}_{\alpha}(L)\right]
$$

We also have:

$$
\sum_{i=1}^{n} w_{i} E\left[D_{i} \mid L \geq \operatorname{VaR}_{\alpha}(L)\right]=\operatorname{ES}_{\alpha}(L)
$$

We remark that the classical risk measure/contribution starting with Markowitz (1952) is based on the standard deviation. Although it remains very popular owing to its computational simplicity, it is less appropriate for credit risk as standard deviation does not properly characterize the tail behavior in the portfolio loss distribution. For more discussions on risk measures and risk contributions we refer the interested reader to Bluhm et al (2002), Denault (2001), Tasche (1999) and Kalkbrener (2005).

\subsection{The Vasicek portfolio credit loss model}

The key issue in portfolio credit loss modeling is the modeling of the default dependence among obligors. It is common practice to utilize the Bernoulli mixture model, such that $D_{i}$ are independent Bernoulli variables conditional on some common factors $Y$ with $\mathbb{P}\left(D_{i}=1 \mid Y\right)=p_{i}(Y)$. The factors $Y$ can represent the state of the economy, different industries and geographical regions, etc.

A broad class of models in the portfolio credit loss modeling can be categorized as Bernoulli mixture models. Examples include all of the popular industrial models such as KMV/Vasicek (Vasicek 2002), CreditRisk ${ }^{+}$(Credit Suisse Financial 
Products 1997) and CreditPortfolioView (Wilson 1997a, b). For more details, see Frey and McNeil (2002, 2003). We concentrate on the Vasicek one-factor Gaussian copula model in the following.

The Vasicek model is a one-period default-mode model, ie, loss only occurs when an obligor defaults in a fixed time horizon. Based on Merton's firm value model, the Vasicek model evaluates the default of an obligor in terms of the evolution of its asset value. Default occurs when the standardized asset log-return $X$ is less than some pre-specified threshold $c$ where $X$ is normally distributed and $\mathbb{P}(X<c)=$ PD. The standardized asset log-return $X$ is decomposed into a systematic part $Y$, representing the state of the economy, and an idiosyncratic part $Z$, such that for obligor $i$ we have:

$$
X_{i}=\sqrt{\rho} Y+\sqrt{1-\rho} Z_{i}
$$

where $Y$ and all $Z_{i}$ are independent and identically distributed (iid) standard normal random variables and $\rho$ is the common pairwise correlation. It is now easily deduced that $X_{i}$ and $X_{j}$ are conditionally independent given the realization of $Y$. This implies that $L_{i}$ and $L_{j}$ are also conditionally independent given $Y$. Further assumptions of the original Vasicek model are that all obligors have the same characteristics, such that $\mathrm{PD}_{i}=p, \mathrm{EAD}_{i}=1$ and $\mathrm{LGD}_{i}=1$, which entails that $w_{i}=1$ for all $i$.

Denote by $p(y)=p_{i}(y)=\mathbb{P}\left[L_{i}=1 \mid Y=y\right]$, ie, the probability of default conditional on the common factor $Y=y$. Then:

$$
p(y)=\mathbb{P}\left[L_{i}=1 \mid Y=y\right]=\mathbb{P}\left[X_{i}<c \mid Y=y\right]=\Phi\left(\frac{\Phi^{-1}(p)-\sqrt{\rho} y}{\sqrt{1-\rho}}\right)
$$

where $\Phi$ is the cumulative distribution function (cdf) of the standard normal distribution.

As a consequence of the strong law of large numbers, one obtains for $n \rightarrow \infty$ :

$$
\mathbb{P}\left[\lim _{n \rightarrow \infty} L / n=p(y) \mid Y=y\right]=1
$$

Equivalently, if we denote by $L(Y)$ the portfolio loss $L$ conditional on $Y$, we have:

$$
\lim _{n \rightarrow \infty} L(Y) / n=p(Y)=\Phi\left(\frac{\Phi^{-1}(p)-\sqrt{\rho} Y}{\sqrt{1-\rho}}\right) \quad \text { almost surely }
$$

Since $p(Y)$ is strictly monotonically decreasing in $Y$, the $\alpha$ quantile of $L$ is simply the $1-\alpha$ quantile of $Y$, ie:

$$
\operatorname{VaR}_{\alpha}=n p\left(\Phi^{-1}(1-\alpha)\right)=n \Phi\left(\frac{\Phi^{-1}(p)+\sqrt{\rho} \Phi^{-1}(\alpha)}{\sqrt{1-\rho}}\right)
$$

As all obligors in the portfolio are equivalent, the VaRC of each obligor is simply $\mathrm{VaR} / n=p\left(\Phi^{-1}(1-\alpha)\right)$.

We note that although the assumptions of uniform pairwise correlation $\rho$ and unconditional default probability PD are made in Vasicek (2002), they are not 
necessary conditions and can be relaxed. Moreover, the convergence in (6) also holds for a portfolio with unequal weights $w_{i}$ if:

$$
\frac{\sum w_{i}^{2}}{\left(\sum w_{i}\right)^{2}} \rightarrow 0
$$

in other words, the portfolio exposure is not concentrated on a few loans that are much larger than the rest. The left-hand side of formula (8) is known as the Herfindahl-Hirschman index (HHI); see, eg, Hirschmann (1964) and Gordy (2003). It provides a simple heuristic approach for quantifying exposure concentration. Well-diversified portfolios with a very large number of very small obligors have a HHI value close to $1 / n$, where $n$ is the number of obligors, whereas heavily concentrated portfolios can have a considerably higher HHI value.

Throughout this paper, the Vasicek model should be considered as a onefactor Gaussian copula model that allows heterogeneous portfolios rather than the restrictive original model for homogeneous portfolios. Summarizing, for a portfolio which is not homogeneous in terms of effective weight, default probability and pairwise correlation, the individual loss variable $L_{i}$ conditional on $Y$ is given by:

$$
L_{i}(Y)= \begin{cases}w_{i} \quad \text { with probability } \Phi\left(\frac{\Phi^{-1}\left(p_{i}\right)-\sqrt{\rho_{i}} Y}{\sqrt{1-\rho_{i}}}\right) \\ 0 \quad \text { with probability } 1-\Phi\left(\frac{\Phi^{-1}\left(p_{i}\right)-\sqrt{\rho_{i}} Y}{\sqrt{1-\rho_{i}}}\right)\end{cases}
$$

If (8) is satisfied, the fraction of loss $\tilde{L}(Y)$ is given by:

$$
\tilde{L}(Y)=\lim _{n \rightarrow+\infty} \frac{\sum_{i=1}^{n} L_{i}(Y)}{\sum_{i=1}^{n} \operatorname{EAD}_{i}}=\frac{\sum_{i=1}^{n} w_{i} \Phi\left(\left(\Phi^{-1}\left(p_{i}\right)-\sqrt{\rho_{i}} Y\right) / \sqrt{1-\rho_{i}}\right)}{\sum_{i=1}^{n} \operatorname{EAD}_{i}}
$$

Then, the VaR and VaRCs are given by:

$$
\begin{gathered}
\operatorname{VaR}_{\alpha}=\sum_{i=1}^{n} w_{i} \Phi\left(\frac{\Phi^{-1}\left(p_{i}\right)+\sqrt{\rho_{i}} \Phi^{-1}(\alpha)}{\sqrt{1-\rho_{i}}}\right) \\
\operatorname{VaRC}_{i, \alpha}=w_{i} \Phi\left(\frac{\Phi^{-1}\left(p_{i}\right)+\sqrt{\rho_{i}} \Phi^{-1}(\alpha)}{\sqrt{1-\rho_{i}}}\right)
\end{gathered}
$$

Note that the VaRC (10) is a portfolio-invariant linear function of $w_{i}$, which implies that the capital contributions of individual exposures only depend on the characteristics of the particular exposure and not on the rest of the portfolio.

The Vasicek asymptotic formula is straightforward, but is heavily reliant on the assumptions of an infinitely large portfolio and of no exposure concentration. When the two conditions, especially the latter, are violated, which constantly occurs in practice, it tends to underestimate risk. Therefore, the analytic formulas are less suitable when a portfolio is of small size or dominated by a few loans much larger than the rest. In the following sections we show that both problems can be handled by the saddlepoint approximation. 


\section{SADDLEPOINT APPROXIMATION}

The computation of the probability distribution function of the sum of independent random variables can be facilitated by the use of the MGF, which is defined by $M_{X}(t)=E\left(\mathrm{e}^{t X}\right)$. For a finite sequence of independent random variables $X_{i}, i=$ $1 \ldots n$, with known analytic MGFs $M_{X_{i}}$, the MGF of the sum $X=\sum_{i=1}^{n} X_{i}$ is the product of MGF of $X_{i}$, ie:

$$
M_{X}(t)=\prod_{i=1}^{n} M_{X_{i}}(t)
$$

Let $K_{X}(t)=\log M_{X}(t)$ be the cumulant generating function (CGF) of $X$. The inverse MGF of $X$ can be written as:

$$
f_{X}(x)=\frac{1}{2 \pi \mathrm{i}} \int_{-\mathrm{i} \infty}^{+\mathrm{i} \infty} \exp \left(K_{X}(t)-t x\right) \mathrm{d} t
$$

Saddlepoint approximation arises in this setting to give an accurate analytic approximation. A detailed exposition of saddlepoint approximations can be found in Jensen (1995). The saddlepoint approximation can be thought of as the Edgeworth expansion at the center of an Esscher transformed density. Only using the approximation at the center of the distribution, the saddlepoint approximation usually leads to a small relative error.

The saddle point, ie, the point at which $K_{X}(t)-t x$ is stationary, is a $t=\tilde{t}$ such that:

$$
K_{X}^{\prime}(\tilde{t})=x
$$

The density $f_{X}(x)$ and the tail probability $\mathbb{P}(X>x)$ can be approximated by $K_{X}(t)$ and its derivative up to second order at $\tilde{t}$.

The Taylor expansion of $K(t)-t x$ (function of $t$ ) around $\tilde{t}$ gives:

$$
K(t)-t x=K(\tilde{t})-\tilde{t} x+\frac{1}{2}(t-\tilde{t})^{2} K^{\prime \prime}(\tilde{t})+\cdots
$$

Substitute (13) into (11), we get:

$$
f_{X}(x) \approx \frac{\exp (K(\tilde{t})-\tilde{t} x)}{2 \pi \mathrm{i}} \int_{-\mathrm{i} \infty}^{+\mathrm{i} \infty} \exp \left(\frac{1}{2}(t-\tilde{t})^{2} K^{\prime \prime}(\tilde{t})\right) \mathrm{d} t=\frac{\exp (K(\tilde{t})-\tilde{t} x)}{\sqrt{2 \pi K^{\prime \prime}(\tilde{t})}}
$$

The tail probability is approximated as:

$$
\begin{aligned}
\mathbb{P}(X>x)= & \frac{1}{2 \pi \mathrm{i}} \int_{-\mathrm{i} \infty,(0+)}^{+\mathrm{i} \infty} \frac{\exp (K(t)-t x)}{t} \mathrm{~d} t \\
& \approx \begin{cases}\exp \left(K(\tilde{t})-\tilde{t} x+\frac{1}{2} \tilde{t}^{2} K^{\prime \prime}(\tilde{t})\right) \Phi\left(-\sqrt{\tilde{t}^{2} K^{\prime \prime}(\tilde{t})}\right) & x>E(X) \\
\frac{1}{2} & x=E(X) \\
1-\exp \left(K(\tilde{t})-\tilde{t} x+\frac{1}{2} \tilde{t}^{2} K^{\prime \prime}(\tilde{t})\right) \Phi\left(-\sqrt{\tilde{t}^{2} K^{\prime \prime}(\tilde{t})}\right) & x<E(X)\end{cases}
\end{aligned}
$$


If all of the $X_{i}$ are identically distributed, the relative errors of both approximations in (14) and (15) are known to be $O\left(n^{-1}\right)$. Higher-order approximations of the density and the tail probability are given by the Daniels (1987) formula:

$$
f_{X}(x)=\frac{\phi\left(z_{l}\right)}{\sqrt{K^{\prime \prime}(\tilde{t})}}\left(\left\{1+\left[-\frac{5 K^{\prime \prime \prime}(\tilde{t})^{2}}{24 K^{\prime \prime}(\tilde{t})^{3}}+\frac{K^{(4)}(\tilde{t})}{8 K^{\prime \prime}(\tilde{t})^{2}}\right]\right\}+O\left(n^{-2}\right)\right)
$$

with $\phi$ the probability density function (pdf) of the standard normal distribution and the Lugannani-Rice (Lugannani and Rice 1980) formula:

$$
\mathbb{P}(X>x)=1-\Phi\left(z_{l}\right)+\phi\left(z_{l}\right)\left[\frac{1}{z_{w}}-\frac{1}{z_{l}}+O\left(n^{-3 / 2}\right)\right]
$$

where $z_{w}=\tilde{t} \sqrt{K^{\prime \prime}(\tilde{t})}$ and $z_{l}=\operatorname{sgn}(\tilde{t}) \sqrt{2[x \tilde{t}-K(\tilde{t})]}$.

\section{SADDLEPOINT APPROXIMATION IN PORTFOLIO CREDIT RISK MODELING}

The saddlepoint approximation method is well known to provide good approximations to very small tail probabilities, which makes it a very suitable technique in the context of portfolio credit loss. The use of saddlepoint approximation in portfolio credit loss was pioneered in a series of articles by Martin et al (2001a, b). Gordy (2002) showed that saddlepoint approximation is fast and robust when applied to CreditRisk ${ }^{+}$. All of these approaches apply saddlepoint approximation to the unconditional MGF of loss $L$, despite the fact that $L_{i}$ are not independent. Annaert et al (2006) showed that the procedure described by Gordy (2002) may give inaccurate results in the case of portfolios with high skewness and kurtosis in exposure size. This paper differs substantially from the cited literature in that we apply the saddlepoint approximation to the conditional MGF of $L$ given the common factor $Y$, so that $L(Y)=\sum w_{i} D_{i}(Y)$ is a weighted sum of independent Bernoulli random variables, which is exactly the situation where the saddlepoint approximation will work well. In Section 5 we show (by a numerical example) that the accuracy of our procedure is not impaired by high skewness and kurtosis in exposure size.

Given that obligors are independent conditional on the common factor, the application of the saddlepoint approximation is straightforward. We write the conditional MGF of $L$ as:

$$
M(t, Y)=\prod_{i=1}^{n}\left(1-p_{i}(Y)+p_{i}(Y) \mathrm{e}^{w_{i} t}\right)
$$

where in the Vasicek model:

$$
p_{i}(Y)=\Phi\left(\frac{\Phi^{-1}\left(p_{i}\right)-\sqrt{\rho_{i}} Y}{\sqrt{1-\rho_{i}}}\right)
$$


The conditional CGF and its derivatives up to fourth order are defined as follows:

$$
\begin{aligned}
K(t, Y)= & \sum_{i=1}^{n} \log \left(1-p_{i}(Y)+p_{i}(Y) \mathrm{e}^{w_{i} t}\right) \\
K^{\prime}(t, Y)= & \sum_{i=1}^{n} \frac{w_{i} p_{i}(Y) \mathrm{e}^{w_{i} t}}{1-p_{i}(Y)+p_{i}(Y) \mathrm{e}^{w_{i} t}} \\
K^{\prime \prime}(t, Y)= & \sum_{i=1}^{n} \frac{\left(1-p_{i}(Y)\right) w_{i}^{2} p_{i}(Y) \mathrm{e}^{w_{i} t}}{\left[1-p_{i}(Y)+p_{i}(Y) \mathrm{e}^{w_{i} t}\right]^{2}} \\
K^{\prime \prime \prime}(t, Y)= & \sum_{i=1}^{n}\left\{\frac{\left(1-p_{i}(Y)\right) w_{i}^{3} p_{i}(Y) \mathrm{e}^{w_{i} t}}{\left[1-p_{i}(Y)+p_{i}(Y) \mathrm{e}^{w_{i} t}\right]^{2}}-\frac{2\left(1-p_{i}(Y)\right) w_{i}^{3} p_{i}^{2}(Y) \mathrm{e}^{2 w_{i} t}}{\left[1-p_{i}(Y)+p_{i}(Y) \mathrm{e}^{w_{i} t}\right]^{3}}\right\} \\
K^{(4)}(t, Y)= & \sum_{i=1}^{n}\left\{\frac{\left(1-p_{i}(Y)\right) w_{i}^{4} p_{i}(Y) \mathrm{e}^{w_{i} t}}{\left[1-p_{i}(Y)+p_{i}(Y) \mathrm{e}^{w_{i} t}\right]^{2}}-\frac{6\left(1-p_{i}(Y)\right) w_{i}^{4} p_{i}^{2}(Y) \mathrm{e}^{2 w_{i} t}}{\left[1-p_{i}(Y)+p_{i}(Y) \mathrm{e}^{w_{i} t}\right]^{3}}\right. \\
& \left.+\frac{\left.6\left(1-p_{i}(Y)\right) w_{i}^{4} p_{i}^{3}(Y) \mathrm{e}^{3 w_{i} t}\right\}}{\left[1-p_{i}(Y)+p_{i}(Y) \mathrm{e}^{w_{i} t}\right]^{4}}\right\}
\end{aligned}
$$

With $K(t, Y)$ available, we are able to calculate the conditional loss density and the conditional tail probability by the saddlepoint approximation. Since $K^{\prime}(t, Y)$ is a monotonically increasing function of $t$ and it is bounded in the interval $\left[0, \sum w_{i}\right]$, the equation $K^{\prime}(t, Y)=x$ admits a unique solution $\tilde{t}$ for $x \in\left[0, \sum w_{i}\right]$. Integrating over $Y$ gives the unconditional loss density and tail probability. For example, the tail probability is given by:

$$
\mathbb{P}(L>x)=\int \mathbb{P}(L>x \mid Y) \mathrm{d} \mathbb{P}(Y)
$$

The VaR can then be found by inverting the loss distribution. Moreover, to obtain the VaRC, we differentiate $\mathbb{P}(L>x)$ with respect to the effective exposure:

$$
\begin{aligned}
& \frac{\partial}{\partial w_{i}} \mathbb{P}(L>x) \\
& \quad=E_{Y}\left\{\frac{1}{2 \pi \mathrm{i}} \int_{-\mathrm{i} \infty,(0+)}^{+\mathrm{i} \infty}\left[\frac{1}{t} \frac{\partial K(t, Y)}{\partial w_{i}}-\frac{\partial x}{\partial w_{i}}\right] \exp (K(t, Y)-t x) \mathrm{d} t\right\}
\end{aligned}
$$

Here we replace $x$ by $\operatorname{VaR}_{\alpha}$. Since the tail probability $\mathbb{P}\left(L>\operatorname{VaR}_{\alpha}\right)$ is fixed at $1-\alpha$, the left-hand side should vanish and we obtain:

$$
\begin{aligned}
& w_{i} \frac{\partial \operatorname{VaR}_{\alpha}}{\partial w_{i}}=w_{i} \frac{E_{Y}\left[\int_{-\mathrm{i} \infty,(0+)}^{+\mathrm{i} \infty}\left(\partial K(t, Y) / \partial w_{i}\right)(1 / t) \exp \left(K(t, Y)-t \mathrm{VaR}_{\alpha}\right) \mathrm{d} t\right]}{E_{Y}\left[\int_{-\mathrm{i} \infty}^{+\mathrm{i} \infty} \exp \left(K(t, Y)-t \mathrm{VaR}_{\alpha}\right) \mathrm{d} t\right]} \\
& =w_{i} \frac{E_{Y}\left[\int_{-\mathrm{i} \infty}^{+\mathrm{i} \infty}\left(p_{i}(Y) \mathrm{e}^{w_{i} t} /\left(1-p_{i}(Y)+p_{i}(Y) \mathrm{e}^{w_{i} t}\right)\right) \exp \left(K(t, Y)-t \mathrm{VaR}_{\alpha}\right) \mathrm{d} t\right]}{E_{Y}\left[f_{L}\left(\mathrm{VaR}_{\alpha} \mid Y\right)\right]}
\end{aligned}
$$


If we define:

$$
\hat{K}^{i}(t, Y)=\log \left(p_{i}(Y) \mathrm{e}^{w_{i} t}\right)+\sum_{j \neq i} \log \left(1-p_{j}(Y)+p_{j}(Y) \mathrm{e}^{w_{j} t}\right)
$$

which can be thought of as the CGF of $L$ given $Y$ and $D_{i}=1$, Equation (27) is rewritten as:

$$
w_{i} \frac{\partial \mathrm{VaR}_{\alpha}}{\partial w_{i}}=w_{i} \frac{E_{Y}\left[\int_{-\mathrm{i} \infty}^{+\mathrm{i} \infty} \exp \left(\hat{K}^{i}(t, Y)-t \mathrm{VaR}_{\alpha}\right) \mathrm{d} t\right]}{E_{Y}\left[f_{L}\left(\mathrm{VaR}_{\alpha} \mid Y\right)\right]}
$$

Both the numerator and the denominator can be approximated by the saddlepoint method.

The VaRC can also be derived in another way. With $\hat{L}^{i}=\sum_{j \neq i} w_{j} D_{j}$, we have:

$$
\begin{aligned}
w_{i} E\left(D_{i} \mid L=\operatorname{VaR}_{\alpha}\right) & =w_{i} \frac{f\left(L=\operatorname{VaR}_{\alpha} ; D_{i}=1\right)}{f_{L}\left(\operatorname{VaR}_{\alpha}\right)} \\
& =w_{i} \frac{E_{Y}\left[f\left(\hat{L}^{i}=\operatorname{VaR}_{\alpha}-w_{i} \mid Y\right) p_{i}(Y)\right]}{E_{Y}\left[f_{L}\left(\operatorname{VaR}_{\alpha} \mid Y\right)\right]}
\end{aligned}
$$

The conditional density in the numerator is the conditional loss density of a portfolio excluding obligor $i$ and can again be calculated by the saddlepoint approximation. We note that (28) and (29) are essentially the same because both formulas use the saddlepoint $\tilde{t}$ that solves:

$$
\sum_{j \neq i} \frac{w_{j} p_{j}(Y) \mathrm{e}^{w_{j} t}}{1-p_{j}(Y)+p_{j}(Y) \mathrm{e}^{w_{j} t}}=\operatorname{VaR}_{\alpha}-w_{i}
$$

Similarly, the ESCs are given by:

$$
w_{i} E\left(D_{i} \mid L \geq \operatorname{VaR}_{\alpha}\right)=w_{i} \frac{E_{Y}\left[\mathbb{P}\left(\hat{L}^{i} \geq \operatorname{VaR}_{\alpha}-w_{i} \mid Y\right) p_{i}(Y)\right]}{E_{Y}\left[\mathbb{P}\left(L \geq \operatorname{VaR}_{\alpha} \mid Y\right)\right]}
$$

and ES can be obtained by simply summing up all of the ESCs, ie:

$$
\mathrm{ES}_{\alpha}=\sum w_{i} E\left(D_{i} \mid L \geq \mathrm{VaR}_{\alpha}\right)
$$

\section{REMARKS}

- Although the obligors in a portfolio are assumed to be completely heterogeneous, for the sake of computational efficiency it is advisable to group obligors as much as possible into homogeneous buckets with similar characteristics, especially for large portfolios. The main advantages of doing this are (i) the expedition of the calculation of conditional CGF and its partial derivatives and (ii) a reduction in the number of risk contributions that need to be computed.

- Martin et al (2001b) proposed a simple estimate to the VaRC, which reads:

$$
\operatorname{VaRC}_{i, \alpha} \approx \frac{E_{Y}\left[\left.f_{L}\left(\operatorname{VaR}_{\alpha} \mid Y\right)\left(w_{i} / \tilde{t}\right)\left(\partial K(t, Y) / \partial w_{i}\right)\right|_{t=\tilde{t}}\right]}{E_{Y}\left[f_{L}\left(\operatorname{VaR}_{\alpha} \mid Y\right)\right]}
$$

in the Bernoulli mixture models. In our numerical examples we show, however, that this approximation may be inaccurate. 


\section{NUMERICAL RESULTS}

\subsection{The Vasicek one-factor model}

We now illustrate the performance of the saddlepoint approximation in the Vasicek one-factor model. For the implementation of the saddlepoint approximation, we always employ the Lugannani-Rice formula (17) for the tail probability. We truncate the common factor $Y$ in the interval $[-5,5]$ so that the probability of $Y$ falling out of this interval is merely $5.7 \times 10^{-7}$. Discretization of $Y$ is done by Gauss-Legendre quadrature, generating 1,000 abscissas and weights. The four examples evaluated are as follows.

- Example 1. A homogeneous portfolio with $n=1,000$ obligors, each with $\mathrm{EAD}=1, \mathrm{LGD}=1, \mathrm{PD}=0.01$ and $\rho=0.2$.

- Example 2. A portfolio consisting of $n=100$ obligors with $\mathrm{EAD}_{i}=i$, $i=1,2, \ldots, 100, \mathrm{PD}=0.1, \rho=0.2$.

- Example 3. A portfolio consisting of one obligor with $\mathrm{EAD}_{1}=100$ and 10,000 obligors with $\mathrm{EAD}_{2}=1$. All obligors have $\mathrm{PD}=0.005$ and $\rho=0.2$.

- Example 4. This portfolio is taken from Glasserman (2006). All 100 obligors have $\mathrm{PD}=0.01$ and $\rho=0.5$. The exposures are:

$$
w_{i}= \begin{cases}1, & i=1, \ldots, 20 \\ 4, & i=21, \ldots, 40 \\ 9, & i=41, \ldots, 60 \\ 16, & i=61, \ldots, 80 \\ 25, & i=81, \ldots, 100\end{cases}
$$

The HHI values for the four portfolios are as follows:

\begin{tabular}{ccccc}
\hline & \multicolumn{4}{c}{ Example } \\
\cline { 2 - 5 } & \multicolumn{1}{c}{$\mathbf{1}$} & \multicolumn{1}{c}{$\mathbf{3}$} & \multicolumn{1}{c}{$\mathbf{4}$} \\
\hline HHI & 0.001 & 0.0133 & 0.0002 & 0.0162 \\
$\frac{1}{n}$ & 0.001 & 0.01 & 0.0001 & 0.01 \\
\hline
\end{tabular}

with $1 / n$ being the HHI value in the case of no exposure concentration.

We compare the loss distribution from the saddlepoint approximation to results from the analytic Vasicek formula and from Monte Carlo simulation in the first two examples. Our benchmark is the sample mean and the accompanying $95 \%$ confidence intervals obtained from 10 subsamples of Monte Carlo simulation with 4 million replications. The loss distribution corresponding to the Vasicek model is obtained by inverting the VaR given by Vasicek's formula (9) for a series of quantile $\alpha$.

Example 1 is an ideal case for the Vasicek formula (9) to be accurate. The loss distributions from different methods are presented in Figure 1(a). The $x$ axis represents the loss percentage, ie, the loss amount in proportion to the 
total exposure. The $y$-axis, the tail probability $P(L>x)$, is in log-scale. It can be seen that both the Vasicek formula and the saddlepoint approximation follow our benchmark very well.

In Example 2, Vasicek's formula significantly underestimates the risk, as is demonstrated in Figure 1(b). This implies the presence of exposure concentration in the portfolio. We observe, however, that the saddlepoint approximation gives results comparable with simulation in this example.

We show more details of the errors made by the saddlepoint approximation for Examples 1 and 2 in Figure 2. Concentrating on the loss percentages from $15 \%$ to $25 \%$, which roughly corresponds to quantiles from $99.9 \%$ to $99.99 \%$ for both examples, we report relative errors compared with the sample means of the 10 subsample estimates. The normalized standard deviations of Monte Carlo simulation with 4 million replications are also provided for comparison. We find that in the tail of the distribution, the relative errors of the saddlepoint approximation are typically smaller than the standard deviations. Furthermore, we see that as loss level increases the standard deviation of Monte Carlo simulation increases significantly, whereas the accuracy of the saddlepoint approximation seems unaffected. This is highly desirable because the tail of the distribution is the center of interest.

Example 3 is a particular test case for which the VaR and VaRCs can be computed almost exactly by the binomial expansion method (BEM) if we treat the portfolio loss as a discrete variable. It is therefore a suitable test portfolio for the calculation of VaRCs. BEM will serve as the benchmark for both the $\mathrm{VaR}$ and the VaRCs. More details on BEM can be found in Appendix A. The loss distributions of this portfolio given by the saddlepoint approximation and the BEM are shown in Figure 3. The saddlepoint approximations again follow our benchmark very well.

As for the VaRC, we first consider a fixed loss level $L=922$, which lies around the $99.9 \%$ quantile. We compute the VaRCs of both the large obligor $\left(\mathrm{VaRC}_{1}\right)$ and any small obligor $\left(\mathrm{VaRC}_{2}\right)$. We use both the standard and higherorder saddlepoint approximation given by (14) and (16), respectively (denoted by SA2 and SA4). Results are shown in Table 1 and the relative errors of the saddlepoint approximation to the benchmark are shown in parentheses. In addition, we compute the Vasicek VaRC, the saddlepoint approximation for the VaRC as given by (33) (denoted by SA-Martin) for comparison.

The results given by the benchmark BEM show that the VaRC increases non-linearly with the size of the exposure. Both the standard and higher-order saddlepoint methods successfully capture this feature and give the VaRCs with small relative errors. The higher-order approximation, with relative error less than $1 \%$, outperforms the standard approximation. The only (negligible) problem is that the VaRCs do not add up to the total VaR exactly. It is also clear that the VaRCs of the large obligor $\left(\mathrm{VaRC}_{1}\right)$ obtained from Vasicek and SA-Martin are both relatively far from the true value. The Vasicek contribution is proportional to the effective exposure and therefore it underestimates the large obligor's risk contribution. SA-Martin penalizes large exposure too much. 
FIGURE 1 Comparison of the Saddlepoint approximation, Vasicek's formula and Monte Carlo simulation for the loss distribution in (a) Example 1 and (b) Example 2. The Monte Carlo 95\% confidence interval (Cl) is constructed using 10 subsamples of 4 million replications each.

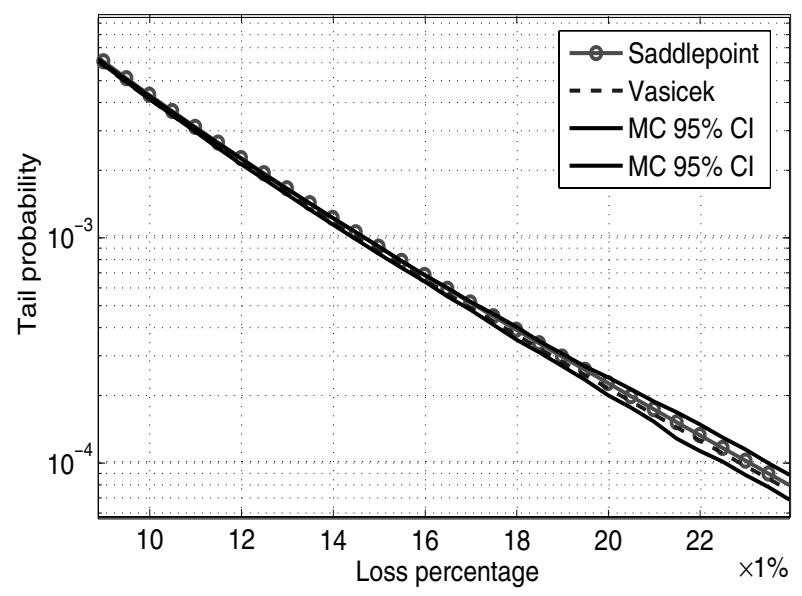

(a)

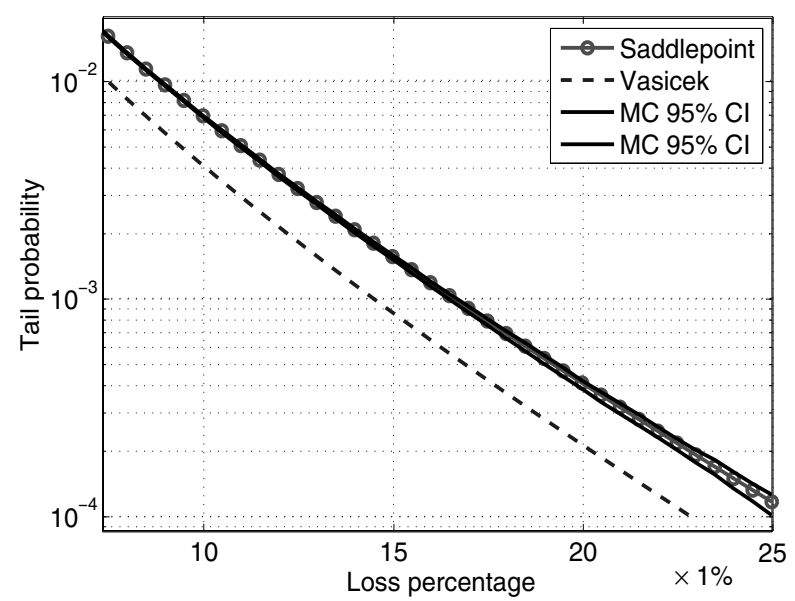

(b)

Next we consider a fixed confidence level $\alpha=99.99 \%$ in Example 3, which is truly far in the tail. The Lugannani-Rice formula is used to compute the loss distribution and the ESCs. The higher-order saddlepoint approximation is used for the VaRCs. Results are shown in Table 2. The accuracy of the saddlepoint approximation is highly satisfactory for all estimates of VaRCs, ESCs and ES. The table suggests that the approximation is slightly more accurate for the VaRC 
FIGURE 2 Comparison of the normalized standard deviations of Monte Carlo simulation with 4 million replications and relative errors of the saddlepoint approximations: (a) Example 1 and (b) Example 2.

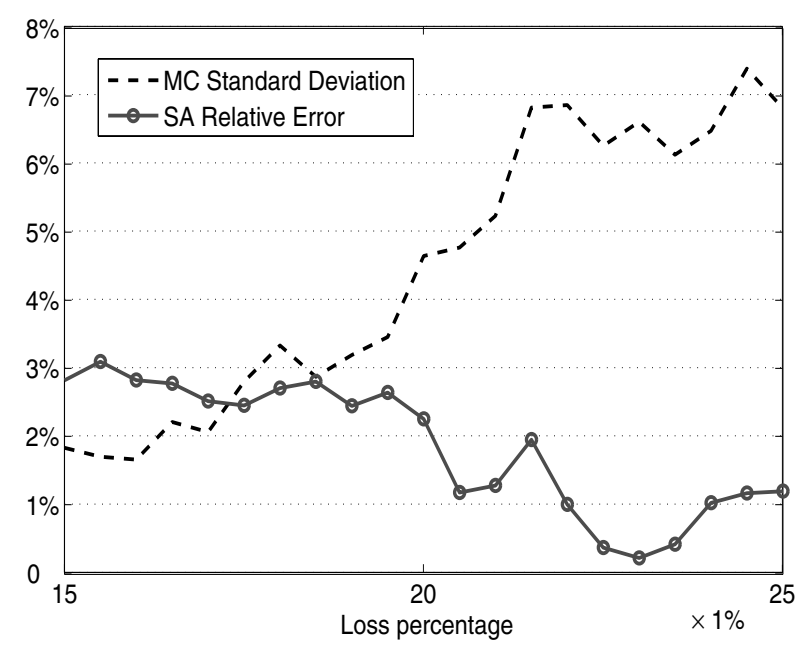

(a)

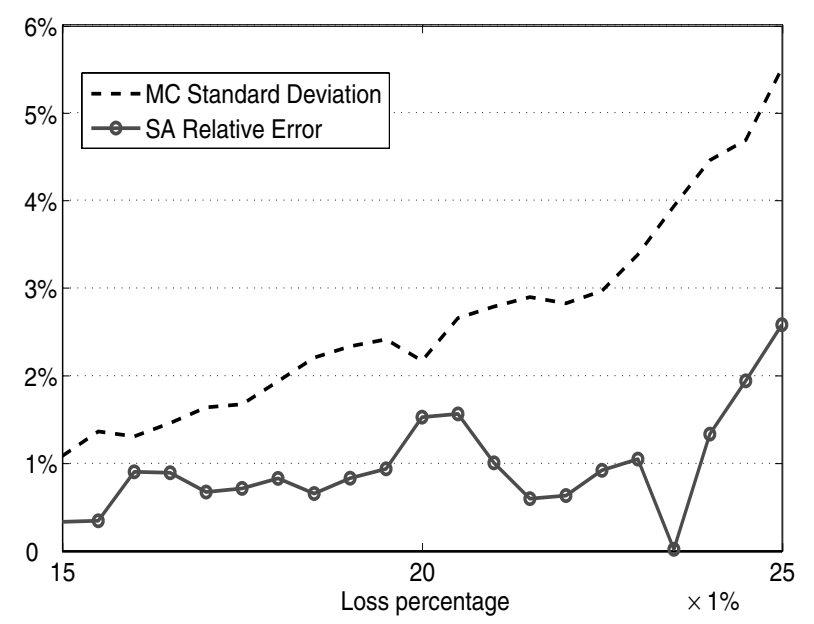

(b)

than for the ESC. This can be understood roughly because the relative error of the Daniels formula is $O\left(n^{-2}\right)$ and that of the Lugannani-Rice formula is $O\left(n^{-3 / 2}\right)$, with $n$ being the number of iid random variables (although in our example $L_{i}$ are not really identically distributed). 
FIGURE 3 Tail probability given by the saddlepoint approximation and the BEM for the portfolio in Example 3.

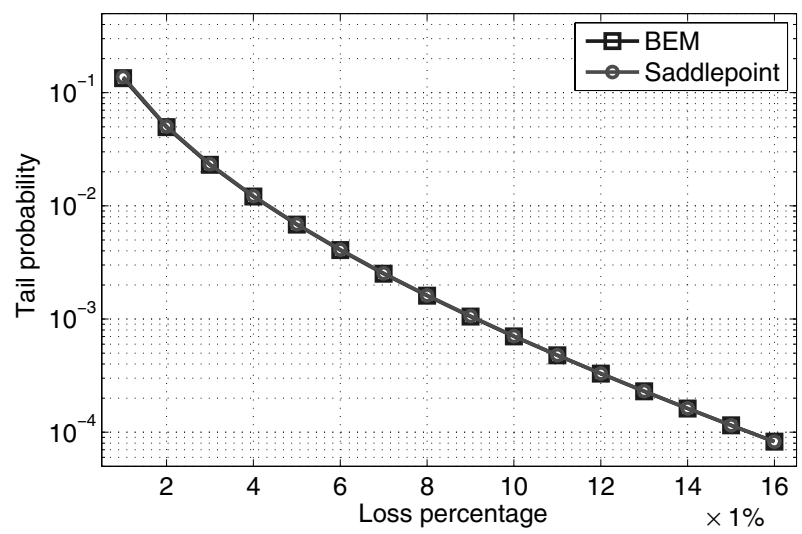

TABLE 1 VaRCs at the loss level $L=922$. The relative errors of the saddlepoint approximation to the benchmark are shown in parentheses. The portfolio is given in Example 3.

\begin{tabular}{llll}
\hline & \multicolumn{1}{c}{ VaRC $_{\mathbf{1}}$} & \multicolumn{1}{c}{ VaRC $_{\mathbf{2}}$} & \multicolumn{1}{c}{$\sum$ VaRC } \\
\hline BEM & 12.61 & 0.0909 & 921.95 \\
Vasicek & 9.13 & 0.0913 & 922 \\
SA-Martin & 21.82 & 0.0900 & 921.99 \\
SA2 & $12.24(2.93 \%)$ & $0.0904(0.55 \%)$ & $916.64(0.58 \%)$ \\
SA4 & $12.65(0.32 \%)$ & $0.0907(0.22 \%)$ & $920.00(0.21 \%)$ \\
\hline
\end{tabular}

TABLE 2 VaRCs and ESCS at the loss level VaR99.99\%. The relative errors of the saddlepoint approximation to the benchmark are shown in parentheses. The portfolio is given in Example 3.

\begin{tabular}{lccccccc}
\hline & VaR99.99\% $_{\text {9aRC }}$ & VaRC $_{\mathbf{1}}$ & $\sum \mathrm{VaRC}$ & $\mathrm{ESC}_{\boldsymbol{1}}$ & $\mathrm{ESC}_{\mathbf{2}}$ & $\mathrm{ES}$ \\
\hline BEM & 1558 & 19.79 & 0.1538 & 1557.87 & 23.14 & 0.1839 & 1862.51 \\
$\mathrm{SA}$ & 1558 & 19.71 & 0.1537 & 1556.27 & 23.18 & 0.1848 & 1871 \\
& & $(0.4 \%)$ & $(0.06 \%)$ & $(0.1 \%)$ & $(0.17 \%)$ & $(0.49 \%)$ & $(0.46 \%)$ \\
\hline
\end{tabular}

We remark that in Example 3 the skewness and kurtosis in exposure size are 99.985 and 9,998, respectively. They are much higher than in portfolios 4 and 5 given in Annaert et al (2006), where it is shown that the accuracy and reliability of the saddlepoint approximation obtained from Gordy's (2002) procedure may deteriorate. In our approach, high skewness and kurtosis do not pose any problem with respect to accuracy.

For the portfolio in Example 4 we report the expected shortfall contributions at the loss level $L=100$. In Glasserman (2006) both VaRC and ESC estimates 
TABLE 3 Comparison of importance sampling (IS) and saddlepoint approximation (SA) for expected shortfall contributions in Example 4 at the loss level $x=100$.

\begin{tabular}{llllll}
\hline & \multicolumn{5}{c}{ Obligors } \\
\cline { 2 - 6 } & $\mathbf{1 - 2 0}$ & $\mathbf{2 1 - 4 0}$ & $\mathbf{4 1 - 6 0}$ & $\mathbf{6 1 - 8 0}$ & $\mathbf{8 1 - 1 0 0}$ \\
\hline IS & 0.10 & 0.42 & 1.02 & 2.03 & 3.67 \\
SA & 0.1017 & 0.4254 & 1.0327 & 2.0453 & 3.6835 \\
\hline
\end{tabular}

from importance sampling are provided. We opt for the latter since the ESC estimates based on simulation are supposedly more accurate and robust: they are conditioned on a relatively less rare event, $\{L \geq 100\}$ compared with $\{L=100\}$ for VaRCs. The results are illustrated in Table 3. Those estimates from importance sampling, using as many as 250,000 replications, are taken from (Glasserman 2006, Table 2) and seen as our benchmark. We observe only marginal differences between ESCs given by the saddlepoint approximation and the benchmark.

It is also interesting to explore the efficiency of the two methods as both appropriately accommodate exposure concentration. Note that both saddlepoint approximation and importance sampling with exponential twisting involves finding the saddlepoint as a solution to (12) for each realization of the common factor. With the saddlepoint at hand, saddlepoint approximation can be obtained analytically, while importance sampling needs to simulate idiosyncratic risks for all obligors in the portfolio. It is more likely than not that the saddlepoint approximation is faster than importance sampling, especially for portfolios with a large number of obligors. In addition, we generally find a draw of 100-1,000 common factors based on the Gauss-Legendre quadrature is sufficient in a onefactor model in terms of accuracy for the saddlepoint approximation, while for importance sampling many more points are necessary to obtain an estimate with small variance, particularly for estimation of the VaRCs. The advantage of importance sampling is that it can compute tail probabilities for a series of loss levels with one set of generated scenarios (see Glasserman and Li 2005). For saddlepoint approximation different saddlepoints need to be found for different loss levels. A final remark is that in all four examples the saddlepoint approximation is able to find the portfolio VaR in less than five seconds on a Pentium $42.8 \mathrm{GHz}$ desktop.

\subsection{Multi-factor model}

We further present an Example 5 under a Gaussian multi-factor model, taken from Glasserman and Li (2005). It is a 21-factor model with $n=1,000$ heterogeneous obligors. The exposures $w_{i}$ increase linearly from 1 to 100 as $i$ increases from 1 to 1,000 . PDs have the following form:

$$
\mathrm{PD}_{i}=0.01 \times(1+\sin (16 \pi i / n)), \quad i=1, \ldots, n
$$


The matrix of factor loading, $A=\left(a_{i j}, i=1, \ldots, 1,000, j=1, \ldots, 21\right)$, has the following block structure:

$$
\mathbf{A}=\left(\begin{array}{c|ccc|c} 
& F & & & G \\
R & & \ddots & & \vdots \\
& & & F & G
\end{array}\right), \quad G=\left(\begin{array}{ccc}
g & & \\
& \ddots & \\
& & g
\end{array}\right)
$$

with: $R$ a column vector of 1,000 entries, all equal to $0.8 ; F$ a column vector of 100 entries, all equal to $0.4 ; G$ a $100 \times 10$ matrix; and $g$ a column vector of 10 entries, all equal to 0.8 .

In a multi-factor model with more than three factors, instead of Gauss quadrature, Monte Carlo simulation or low-discrepancy sequences can be employed for the integration. The saddlepoint approximation itself, however, is unaffected, since all of the information of the common factors is encapsulated in $p_{i}(Y)$ before starting the approximation procedure. We note that when $x$ is large and $Y$ is large and positive, $\mathbb{P}(L>x \mid Y)$ will tend to zero and thus the integration in (25) will not be efficient. It is a natural idea to resort to importance sampling for a significant improvement in such cases. By choosing a $\mathbb{P}$-equivalent probability measure $\mathbb{Q}$, the tail probability can be rewritten as:

$$
\mathbb{P}(L>x)=\int \mathbb{P}(L>x \mid Y) \mathrm{d} \mathbb{P}(Y)=\int \mathbb{P}(L>x \mid Y) \frac{\mathrm{d} \mathbb{P}(Y)}{\mathrm{d} \mathbb{Q}(Y)} \mathrm{d} \mathbb{Q}(Y)
$$

Several procedures to find the optimal measure $\mathbb{Q}$ have been suggested by Glasserman and Li (2005) and Glasserman (2006).

A hybrid method of saddlepoint approximation and importance sampling is more efficient than pure importance sampling, since in the simulation only the common factors need to be generated and not the idiosyncratic risks. This is more advantageous for large portfolios. Moreover, for the calculation of the VaRC importance sampling can only use the few replications $L=x$, whereas the hybrid method need not condition on this rare event.

We employ the hybrid method for Example 5. It is carried out as follows. Taking the same mean shift in the common factors as in Glasserman and $\mathrm{Li}$ (2005), ie, 2.46 for the first factor and 0.2 for the other components, we generate 10 subsamples of simulation with 1,000 replications each. The saddlepoint approximation is employed to compute the tail probability conditional on each realization of the common factors. Afterwards the conditional tail probabilities are aggregated with proper likelihood ratios. Table 4 shows the resulting tail probabilities and their standard deviations for five loss levels from 10,000 to 30,000 , with reference to those point estimates reported in Glasserman and $\mathrm{Li}$ (2005), which are based on pure importance sampling. Differences between the two methods appear to be immaterial. In addition, we produce a column of standard deviations of pure importance sampling with $10 \times 1,000$ replications alongside the point estimates. It can immediately be seen that the hybrid method yields smaller standard deviations. The larger variance associated to the pure importance sampling method can be attributed to variations in the idiosyncratic risks. 
TABLE 4 Comparison of importance sampling (IS) and the hybrid method for point estimates and standard deviations of tail probability $P(L>x)$ at various loss levels. The portfolio is given in Example 5.

\begin{tabular}{ccccc}
\hline $\boldsymbol{x}$ & IS & Standard deviation & Hybrid & Standard deviation \\
\hline 10,000 & 0.0114 & $8.17 \times 10^{-4}$ & 0.01139 & $4.55 \times 10^{-4}$ \\
14,000 & 0.0065 & $3.85 \times 10^{-4}$ & 0.00641 & $3.41 \times 10^{-4}$ \\
18,000 & 0.0037 & $2.57 \times 10^{-4}$ & 0.00367 & $1.49 \times 10^{-4}$ \\
22,000 & 0.0021 & $1.89 \times 10^{-4}$ & 0.00210 & $8.12 \times 10^{-5}$ \\
30,000 & 0.0006 & $6.53 \times 10^{-5}$ & 0.00063 & $4.81 \times 10^{-5}$ \\
\hline
\end{tabular}

\section{FURTHER EXTENSIONS}

Although we confine our numerical experiments to the Gaussian factor models in previous section, the saddlepoint approximation technique can be readily applied to all Bernoulli mixture models with common factors that follow any distribution. Recall that in our approach the distribution of portfolio loss $L$ is obtained by integrating the conditional distribution of $L(Y)$ and the saddlepoint approximation only deals with $L(Y)$. A different choice of mixture model gives a difference in the form of the conditional default probability $p_{i}(Y)$, eg, in the Vasicek one-factor model $p_{i}(Y)$ is given by (19) and in CreditRisk ${ }^{+}$:

$$
p_{i}(Y)=p_{i}\left(w_{i 0}+\sum Y_{k} w_{i k}\right)
$$

where $Y_{k}$ are assumed to be independently gamma distributed (see Gordy 2002). However, then the conditional portfolio loss $L(Y)=\sum w_{i} D_{i}(Y)$ reduces to a weighted sum of independent Bernoulli random variables, whose MGF always exists. As the main requirement of calculating a saddlepoint approximation is the existence of a MGF, the saddlepoint approximation is applicable in any Bernoulli mixture model.

Furthermore the saddlepoint approximation can also handle LGD volatility. When the LGD, which was assumed to be constant, becomes a random variable, the conditional CGF reads:

$$
K(t, Y)=\sum \log \left[1-p_{i}(Y)+p_{i}(Y) E\left(\mathrm{e}^{w_{i} t} \mid Y\right)\right]
$$

Various forms of distribution of LGD can be found in the literature. For example, in Frye's (2000) model, the LGD is modeled as a normal random variable with mean $\mu$ and standard deviation $\sigma$ such that:

$$
\operatorname{LGD}_{i}=\mu+\sigma\left(-b_{i} Y+\sqrt{1-b_{i}^{2}} \epsilon_{i}\right)
$$

Here the $\epsilon_{i}$, independent of $Y$, are assumed to be iid standard normal variables and the $b_{i}$ are assumed to be positive to insure the correct qualitative effect of LGD, which is mostly determined by the value of collateral. It should tend to be higher 
when the economy is weak and lower when the economy is strong. It follows that:

$$
\begin{aligned}
E\left(\mathrm{e}^{w_{i} t} \mid Y\right) & =\mathrm{e}^{\operatorname{EAD}_{i}\left(\mu-\sigma b_{i} Y\right) t} E\left(\mathrm{e}^{\operatorname{EAD}_{i} \sigma \sqrt{1-b_{i}^{2}} \epsilon_{i} t}\right) \\
& =\exp \left(\operatorname{EAD}_{i}\left(\mu-\sigma b_{i} Y\right) t+\operatorname{EAD}_{i}^{2} \sigma^{2}\left(1-b_{i}^{2}\right) t^{2} / 2\right)
\end{aligned}
$$

After substitution of (35) into (34), we see that a random LGD will not complicate the problem further.

\section{CONCLUSIONS}

We have described a new procedure to embed the saddlepoint approximation as a useful tool in portfolio credit loss modeling. We apply the saddlepoint approximation in the Vasicek one-factor model. The saddlepoint approximations, especially the higher-order approximations, are able to produce accurate results on both the VaR and the VaRC. The ES and ESC can also be computed satisfactorily. We have also illustrated that the saddlepoint approximation works well for small portfolios and portfolios with exposure concentration, where Vasicek's asymptotic formulas fail. We further point out that the saddlepoint approximation is a flexible method that can be applied in quite general situations, for example, in other Bernoulli mixture (possibly multi-factor) models and portfolios with random LGD.

\section{APPENDIX A BINOMIAL EXPANSION METHOD}

The BEM is similar to the recursive method proposed by Andersen et al (2003) in the sense that both methods treat the portfolio loss as a discrete variable. The former method is tailor-made for the portfolio in Example 3 we considered in Section 4 , while the latter method can be applied to more general portfolios. An evaluation of the recursive method can be found in Glasserman and Ruiz-Mata (2006).

Consider a portfolio consisting of one obligor with $\mathrm{EAD}_{1}=k, \mathrm{PD}=p_{1}$ and $n$ obligors with $\mathrm{EAD}_{2}=1, \mathrm{PD}=p_{2}$. In a Bernoulli mixture model, the losses of the obligors are conditionally independent given the common factor $Y$. Let $p_{1}(Y)$ and $p_{2}(Y)$ be the conditional default probabilities, we have:

$$
\begin{aligned}
\mathbb{P}(L=m) & =\int \mathbb{P}(L=m \mid Y) \mathrm{d} \mathbb{P}(Y) \\
& =\int p_{1}(Y) \mathbb{P}\left(L^{n}=m-k \mid Y\right)+\left(1-p_{1}(Y)\right) \mathbb{P}\left(L^{n}=m \mid Y\right) \mathrm{d} \mathbb{P}(Y)
\end{aligned}
$$

where:

$$
\mathbb{P}\left(L^{n}=m \mid Y\right)=\left(\begin{array}{l}
n \\
m
\end{array}\right)\left(p_{2}(Y)\right)^{m}\left(1-p_{2}(Y)\right)^{n-m}
$$

The VaR and VaRCs are then given, respectively, by:

$$
\operatorname{VaR}_{\alpha}=\inf \left\{x \mid \sum_{m=0}^{x} \mathbb{P}(L=m) \geq \alpha\right\}
$$


and:

$$
\begin{aligned}
& \operatorname{VaRC}_{1}=\frac{\int p_{1}(Y) \mathbb{P}\left(L^{n}=\operatorname{VaR}_{\alpha}-k \mid Y\right) \mathrm{d} \mathbb{P}(Y)}{\mathbb{P}\left(L=\operatorname{VaR}_{\alpha}\right)} \\
\operatorname{VaRC}_{2}= & \frac{1}{\mathbb{P}\left(L=\operatorname{VaR}_{\alpha}\right)} \\
& \times\left\{\int p_{2}(Y) p_{1}(Y) \mathbb{P}\left(L^{n-1}=\operatorname{VaR}_{\alpha}-k-1 \mid Y\right) \mathrm{d} \mathbb{P}(Y)\right. \\
& \left.+\int p_{2}(Y)\left(1-p_{1}(Y)\right) \mathbb{P}\left(L^{n-1}=\operatorname{VaR}_{\alpha}-1 \mid Y\right) \mathrm{d} \mathbb{P}(Y)\right\}
\end{aligned}
$$

The ESCs are computed according to (31) with:

$$
\mathbb{P}(L \geq x)=1-\sum_{m=0}^{x-1} \mathbb{P}(L=m)
$$

and ES is obtained by (32).

\section{REFERENCES}

Andersen, L., Sidenius, J., and Basu, S. (2003). All your hedges in one basket. RISK (November), 67-72.

Annaert, J., Garcia, J. B. C., Lamoot, J., and Lanine, G. (2006). Don't fall from the saddle: the importance of higher moments of credit loss distributions. Technical Report 06/367, Faculty of Economics and Business Administration, Ghent University, Belgium.

Artzner, P., Delbaen, F., Eber, J.-M., and Heath, D. (1999). Coherent measures of risk. Mathematical Finance 9(3), 203-228.

Basel Committee on Bank Supervision (2005). Basel II: International Convergence of Capital Measurement and Capital Standards: A Revised Framework. BCBS Publications No. 118, Bank for International Settlements, Basel.

Bluhm, C., Overbeck, L., and Wagner, C. (2002). An Introduction to Credit Risk Modeling. Chapman \& Hall/CRC, Boca Raton, FL.

Credit Suisse Financial Products (1997). CreditRisk ${ }^{+}$: a CreditRisk management framework, Credit Suisse Financial Products.

Daniels, H. (1987). Tail probability approximations. International Statistical Review 55, 37-48.

Denault, M. (2001). Coherent allocation of risk capital. Journal of Risk 4(1), 7-21.

Frey, R. and McNeil, A. J. (2002). VaR and expected shortfall in portfolios of dependent credit risks: conceptual and practical insights. Journal of Banking and Finance 26, 1317-1334.

Frey, R., and McNeil, A. J. (2003). Dependent defaults in models of portfolio credit risk. Journal of Risk 6(1), 59-92.

Frye, J. (2000). Depressing recoveries. RISK (November), 108-111.

Glasserman, P. (2006). Measuring marginal risk contributions in credit portfolios. Journal of Computational Finance $\mathbf{9}(2), 1-41$.

Journal of Computational Finance

Volume 11/Number 1, Fall 2007 
Glasserman, P., and Li, J. (2005). Importance sampling for credit portfolios. Management Science 51(11), 1643-1656.

Glasserman, P., and Ruiz-Mata, J. (2006). Computing the credit loss distribution in the Gaussian copula model: a comparison of methods. Journal of Credit Risk 2(4), 33-66.

Gordy, M. B. (2002). Saddlepoint approximation of CreditRisk ${ }^{+}$. Journal of Banking and Finance 26, 1335-1353.

Gordy, M. B. (2003). A risk-factor model foundation for ratings-based bank capital rules. Journal of Financial Intermediation 12(3), 199-232.

Gourieroux, C., Laurent, J.-P., and Scaillet, O. (2000). Sensitivity analysis of values at risk. Journal of Empirical Finance 7, 225-245.

Hirschmann, A. O. (1964). The paternity of an index. American Economic Review 54, 761-762.

Jensen, J. (1995). Saddlepoint Approximations. Oxford University Press, Oxford.

Kalkbrener, M. (2005). An axiomatic approach to capital allocation. Mathematical Finance 15(3), 425-437.

Lugannani, R., and Rice, S. (1980). Saddlepoint approximations for the distribution of the sum of independent random variables. Advances in Applied Probability 12, 475-490.

Markowitz, H. M. (1952). Portfolio selection. Journal of Finance 7(1), 77-91.

Martin, R., Thompson, K., and Browne, C. (2001a). Taking to the saddle. RISK (June), 91-94.

Martin, R., Thompson, K., and Browne, C. (2001b). VAR: who contributes and how much? RISK (August), 99-102.

Tasche, D. (1999). Risk contributions and performance measurement. Technical Report, Technische Universitat Muenchen.

Vasicek, O. (2002). Loan portfolio value. RISK (December), 160-162.

Wilson, T. (1997a). Portfolio credit risk I. RISK (September), 111-117.

Wilson, T. (1997b). Portfolio credit risk II. RISK (October), 56-61. 